\title{
HIGH ENERGY PHYSICS IN MAGNETIC JETS AND HOT SPOTS
}

\author{
Peter L. BIERMANN \\ Max-Planck-Institut für Radioastronomie \\ Auf dem Hügel 69 \\ 5300 Bonn 1, F.R.G.
}

ABSTRACT. In this progress-report I wish to describe the work my scientific collaborators and I have done over the last six years.

In 1976 Rieke et al. noticed for the first time that some active nuclei had a sharp cutoff in their nonthermal emission in the near infrared to red wavelength range. Over the following years it became obvious mostly through the work of groups in Tucson, Tenerife and Heidelberg that this is a widespread phenomenon in red quasars, in BL Lac's and in hot spots as well as jets.

After initial attempts (Webb et al. 1984, Schlickeiser 1984) Biermann and Strittmatter (1987) proposed a theory to explain the observations described above: The theory is based on the idea that first order Fermi acceleration takes place in shocks, in hot spots, knots in jets, and nuclear emission regions of active nuclei. Klemens (1987) investigated the role of proton-proton collisions as sources of relativistic electronpositron pairs to generate $X$-ray synchrotron emission to explain the $X$-ray emission of the M87 jet. Fritz (1987, 1989a,b) investigated in some detail the shape of the cutoff for different assumptions about the spectrum of the turbulence, in his latest paper introducing the non-linear effects of self-excited turbulence. Balancing adiabatic losses with acceleration rather than synchrotron losses, Völk and Biermann (1988) were able to explain the high energies of the particles at the spectral knee of cosmic rays, applying similar ideas as those developed for quasars to supernova explosions in stellar wind cavities.

Following Kirk and Schneider, Krülls (1989) is now looking at cutoff spectra in relativistic shocks, while Fraix-Burnet $(1988,1989 a, b, c)$ from the group of J.-L. Nieto in close contact with us is considering oblique shocks as an important ingredient. The requirement of first-order Fermi acceleration puts strong constraints on the shock-magnetic field configuration.

K. Mannheim and I have recently started to consider the details of the high energy proton-photon interactions and their consequences for the $x$-ray, $y$-ray and neutrino spectra of active nuclei (Mannheim and Biermann, 1989); critical is the initial maximum energy of heavy nuclei, dependent on the magnetic field. 


\section{References}

Biermann, P.L. and Strittmatter, P.A. (1987) Astrophys. J. 322, 643.

Fraix-Burnet, D. and Nieto, J.L. (1988) Astron. Astrophys. 198, 87.

Fraix-Burnet, D., Nieto, J.-L., Lelièvre, G., Macchetto, F.D., Perryman,

M.A.C. and di Serego Alighieri, S. (1989a) Astrophys. J. 336, 121.

Fraix-Burnet, D. (1989b) Ph.D. Thesis, Univ. Toulouse.

Fraix-Burnet, D. (1989c) Astron. Astrophys. (in press).

Fritz, K.-D. (1987) Ph.D. Thesis, Univ. Bonn.

Fritz, K.-D. (1989a) Astron. Astrophys. 214, 14.

Fritz, K.-D. (1989b) Astrophys. J. (in press).

Klemens, Y. (1987) Ph.D. Thesis, Univ. Bonn.

Krülls, W. (1989) Ph.D. Thesis, Univ. Bonn.

Mannheim, K. and Biermann, P.L. (1989) Astron. Astrophys. (in press).

Schlickeiser, R. (1984) Astron. Astrophys. 136, 227.

Völk, H.-J. and Biermann, P.L. (1988) Astrophys. J. Letters 333, L65.

Webb, G.M., Drury, L.O'C and Biermann, P.L. (1984) Astron. Astrophys. 137, 185. 